\title{
Based on ECM Modelling for Daily Turnover and Close Index of Chinese Stock Markets
}

\author{
Xiaohua $\mathrm{Hu}^{1} \&$ Min $\mathrm{Yu}^{2}$ \\ ${ }^{1}$ School of Mathematics and Statistics, Hainan Normal University, Haikou, China \\ ${ }^{2}$ Dean's Office of Hainan Normal University, Haikou, China \\ Correspondence: Xiaohua Hu, School of Mathematics and Statistics, Hainan Normal University, Haikou 571158, \\ China. Tel: 86-577-65909079. E-mail: 1241957415@qq.com
}

Received: August 20, 2012

Accepted: September 16, 2012

Online Published: September 14, 2012

doi:10.5539/ijef.v4n11p205

URL: http://dx.doi.org/10.5539/ijef.v4n11p205

\begin{abstract}
By making use of test for stationary, Granger, co-integration, we study the daily turnover and daily close index of Chinese stock markets from 1991 to 2011. We strive to find how Shanghai and Shenzhen stock markets interact each other, there really exist a long-run equilibrium equation among the daily close index,daily turnover of Shanghai (Shenzhen) market and daily close index of Shenzhen (Shanghai) market, to establish the two-order bivariate error correction model(ECM)for two Chinese stock markets respectively. We also further analyze the act of the fluctuation of daily close index of the two markets in short-term.
\end{abstract}

Keywords: turnover, close index, ECM, co-integration, Granger cause

\section{Introducation}

Shanghai stock market and Shenzhen stock market, both established in the early 1990s, sometimes are affected by the western stock markets.Due to Chinese special financial policy, however the development tendency of two Chinese markets has its own character every day, that is when the index of one market goes up, the index of another market not necessarily goes up even goes down in their early period of development. The phenomena of "Shanghai market gets strong and Shenzhen market becomes weak",or vice versa occurred from time to time, one market's development change is often different from another one's every day, two stock markets are almost independent of each other.However,as time passes, the interaction between Shanghai stock market and Shenzhen stock market has been becoming more and more obvious. The phenomena of "Shanghai (Shenzhen) market strong and Shenzhen(Shanghai) market weak" is no longer the case nowadays. Two Chinese stock markets seemingly rise up or drop down almost at the same time,

The relation between business volume and price in stock market is of interests to the professional people.In general, increase in business amount causes index climing up, decline in business amount causes index down. There are a lot of literatures that research the relation between the close index and the turnover in the same market. For example,in the recent papers, ZHOU Xiao-yan(2012) studied the volume-price relation in Shenzhen Stock Market by using impulse response function and variance decomposition from 2003 to 2006. ZHAI Ai-mei\&ZHOU Tong(2011) analyzed the volume-price relation in stockmarket from 2006 to 2010 by using Behavioral Finance. GUO Liang \& ZHOU Weixing (2010) performed an empirical analysis of the volume-price relation in the Chinese stock market at microscopic level using high-frequency data from January to June 2006. TONG Menghua\& WU Chengming (2009) studied the dynamic relation between volume and price in Shanghai stock market based on the CARR model from 1999 to 2007 etc.

These papers all have focused on the single stcoket market, they have not considered the interaction between two markets. Moreover, Sample data used in their research is limited, and the time range of sample data is finite. In this paper,we expand the time range of sample data from 1991 to 2011, and consider the interaction between two markets, by the use of mainly bivariate ECM to study the volume- price relation. We noticed that In Chinese stock markets, the index of one market is affected not only by its own turnover but also by another market index, but the index of one market is irrelevant with the turnover of another market, although the close index and turnover appears occurring in a random way every day. There likely exists a long-run equilibrium relation among the daily close index, daily turnover in same market and daily close index of another market. The question 
arising is What is their relation in long-term? Furthermore, what does cause the fluctuation of the close index in one market in short-term? These problems are what we are concerned and what we intend to study in this paper, the answer to these problems are very important for investors in the stock markets. Hopefully the findings from this study provide investors some helpful advices to lower risk and improve return.

The reminder paper is organized as follows. Section 2 presents the method used in this paper. Section 3 presents the empirical analysis and modelling. The final section presents conclusions.

\section{Methodology}

There are lots of papers to introduce how to test the stationary of the variables using the Augmented Dickey-Fuller (ADF), how to test for causality from variable A to variable B (and variable B to variable A) using the Granger and how to test, provided the co-integration has been found, co-integration between variables using the Johansen (or others) technique. In this paper, we mainly introduce bivariate ECM.

Suppose that there is a long-run equilibrium relation among variables $Y_{t}, X_{t}$ and $Z_{t}$ as below

$$
Y_{t}=\alpha_{0}+\alpha_{1} X_{t}+\alpha_{2} Z_{t}+u_{t}
$$

However, actually variable $Y_{t}, X_{t}$ and $Z_{t}$ seldom exist the long-run equilibrium relation at $t$ moment.Let $e c m_{t}=\mu_{t}=Y_{t}-\alpha_{0}-\alpha_{1} X_{t}-\alpha_{2} Z_{t}$, we then can establish multiple variables two-order error correction model (ECM) as below.

$$
\Delta Y_{t}=\lambda e v m_{t-1}+\beta_{1} \Delta X_{t}+\beta_{2} \Delta X_{t-1}+\gamma_{1} \Delta Z_{t}+\gamma_{2} \Delta Z_{t-1}+\varepsilon_{t}
$$

Where $\varepsilon_{t}$ is white-noise series and $\alpha_{0}, \alpha_{1}, \alpha_{2}, \lambda, \beta_{1}, \beta_{2}, \gamma_{1}, \gamma_{2}$ are parameters to be estimated.

\section{Empirical Analysis and Modelling}

\subsection{Data and Variables}

The time range of sample data is from $3 / 4 / 1991$ to $28 / 11 / 2011$, hence the total size of sample data is 5043 , Let $x_{1 t}, x_{2 t}(t=1,2, \ldots, 5042)$ represent the daily close index, daily turnover of Shanghai market at the $t-t h$ day respectively, whilist $y_{1 t}, y_{2 t}(t=1,2, \ldots, 5042)$ be the daily close index,daily turnover of Shenzhen market at the $t-t h$ day respectively. We also set $l x_{1 t}=\ln x_{1 t}, l x_{2 t}=\ln x_{2 t}, l y_{1 t}=\ln y_{1 t}, l y_{2 t}=\ln y_{2 t}$.

\subsection{Test for Stationary and Granger}

We find $l x_{i t}, l y_{i t}(i=1,2)$ are not stationary by test for unit root, though they are stationary by one-difference, or, $l x_{i t}, l y_{i t}(i=1,2)$ are $I(1)$, For $l x_{i t}, l y_{i t}(i=1,2)$, according to "AIC",we take lags to include $k=1$, then we tabulate the test for Granger in table 1 below.

\begin{tabular}{|c|c|c|c|c|c|}
\hline Null & Hypothesis & & Obs & F-Statistic & Prob. \\
\hline$\Delta l x_{2 t}$ & does not Granger Cause & $\Delta l x_{1 t}$ & 5040 & 1.64919 & 0.1991 \\
\hline$\Delta l x_{1 t}$ & does not Granger Cause & $\Delta l x_{2 t}$ & & 194.471 & $2.00 \mathrm{E}-43$ \\
\hline$\Delta l y_{2 t}$ & does not Granger Cause & $\Delta l y_{1 t}^{2 t}$ & & 1.03545 & 0.3089 \\
\hline$\Delta l y_{1 t}$ & does not Granger Cause & $\Delta l y_{2 t}$ & & 257.246 & $2.00 \mathrm{E}-56$ \\
\hline$\Delta l y_{1 t}$ & does not Granger Cause & $\Delta l x_{1 t}$ & & 0.54933 & 0.4586 \\
\hline$\Delta l x_{1 t}$ & does not Granger Cause & $\Delta l y_{1 t}$ & & 0.10067 & 0.751 \\
\hline
\end{tabular}

Table 1. Test for Granger

Whether the significance level is $1 \%, 5 \%$ or $10 \%$, we accept $\Delta l x_{2 t}$ Granger causes $\Delta l x_{1 t}, \Delta l y_{2 t}$ Granger causes $\Delta y_{1 t}, \Delta l x_{1 t}$ Granger causes $\Delta l y_{1 t}, \Delta l y_{1 t}$ Granger causes $\Delta l x_{1 t}$. 


\subsection{Test for Co-integration}

We establish the regression equations as below

$$
\begin{aligned}
& l x_{1 t}=0.11431 l x_{2 t}+0.562769 l y_{1 t}+u_{t} \\
& l y_{1 t}=0.060579 l y_{2 t}+0.955076 l x_{1 t}+v_{t}
\end{aligned}
$$

Where $u_{t}, v_{t}$ are random errors, note in (3) $R^{2}=0.917$, D.W=0.047, in (4) $R^{2}=0.871$, D.W $=0.010$. We perform unit root test for $u_{t}$, then generate some results given in table 2 below

Table 2. Augmented Dickey-Fuller (ADF) test for $u_{t}$

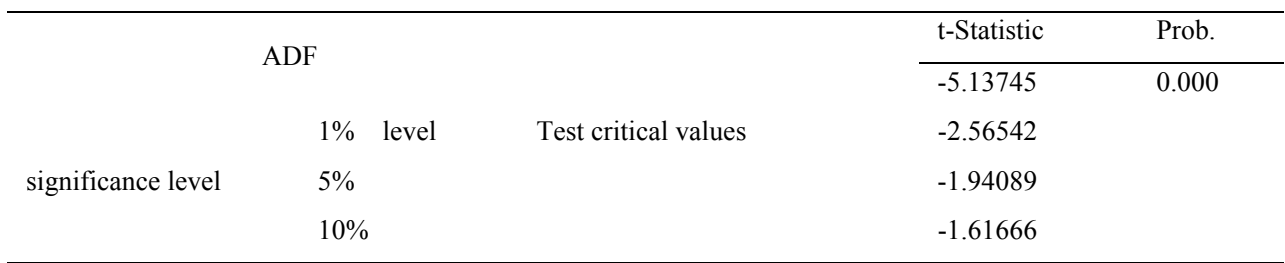

In same way, we can perform unit root test for $v_{t}$, and obtain t-Statistic=-5.388414. It is not hard to see $u_{t} \square$ $I(0), v_{t} \square I(0)$. These observations show that there exists an co-integration relation among $l x_{1 t}, l x_{2 t}$ and $l y_{1 t}$, an co-integration relation among $l y_{1 t}, l y_{2 t}$ and $l x_{1 t}$, from $3 / 4 / 1991$ to $28 / 11 / 2011$, where the co-integration vector are $(1,-0.114310,-0.562769),(1,-0.060579,-0.955076)$, respectively.

\subsection{Modeling for ECM}

Now in turn, we consider the long-run equilibrium equation among the daily close index $l x_{1 t}$, daily turnover $l x_{2 t}$ of Shanghai market and daily close index $l y_{1 t}$ of Shenzheng market. Firstly let's establish the equations as below

$$
\begin{aligned}
& l x_{1 t}=0.54296+0.1179761 l x_{2 t}+0.4873571 l y_{1 t}+u_{t} \\
& l y_{1 t}=0.678149+0.064201 l y_{2 t}+0.850619 l x_{1 t}+v_{t}
\end{aligned}
$$

Notice that In (5), $R^{2}=0.92$, D.W=0.053, in (6), $R^{2}=0.90$, D.W=0.02, we take the residual series $u_{t}, v_{t}$ to be the error correction term. Accordingly, we establish two-order Error Correction Model (ECM) for $l x_{1 t}$ and $l y_{1 t}$, respectively.

$$
\begin{gathered}
\Delta l x_{1 t}=-0.00761 e \mathrm{em}_{t-1}+0.0085 \Delta l x_{2 t}+0.0023 \Delta l x_{2 t-1}+0.691 \Delta l y_{1 t}-0.0282 \Delta l y_{1 t-1}+\varepsilon_{t} \\
\Delta l y_{1 t}=-0.0038 e c m_{t-1}+0.01 \Delta l y_{2 t}+0.0023 \Delta l y_{2 t-1}+0.551 \Delta l x_{1 t}+\varepsilon_{t}
\end{gathered}
$$

Note In (7), $R^{2}=0.43$, D.W=1.983, but In (8), $R^{2}=0.436$, D.W=1.98, (7) and (8) show $\varepsilon_{t}$ doesn't exist one-order autocorrelation, coefficient of Autocorrelation and Partial Correlation further show $\varepsilon_{t}$ don't exist high-order autocorrelation..But it shows that the significance test for coefficients in (7), (8) and regression equation (7), (8) can pass whether the significance level is $1 \%, 5 \%$ or $10 \%$.

\section{Conclusion}

By Test for stationary,Granger,co-integration, we find that there really exist the long-run equilibrium equation among the daily close index,daily turnover of Shanghai (Shenzhen) market and daily close index of Shenzhen (Shanghai) market.

In the long run, by (5), we noticed that $x_{1 t}$ will change $0.1179761 \%$ when $x_{2 t}$ changes $1 \%$ but $y_{1 t}$ keeps unchanged, while $x_{1 t}$ will change $0.4873571 \%$ when $y_{1 t}$ changes $1 \%$ but $x_{2 t}$ keeps unchanged; Similarily by 
(6), $y_{1 t}$ will change $0.064201 \%$ when $y_{2 t}$ changes $1 \%$ but $x_{1 t}$ keeps unchanged, $y_{1 t}$ will change $0.850619 \%$ when $x_{1 t}$ changes $1 \%$ but $y_{2 t}$ keeps unchanged.

In the short run,by (7) and (8), the fluctuation of the close index of Shanghai(Shenzhen) stock market was caused by two factors.The first one was the effect of the close index deviating from long-run equilibrium.In particular the size of coefficient of term ecm ef-1 $_{1}$ reflects adjusting strength of deviating from long-run equilibrium, the estimation value of coefficient $-0.00761(-0.0038)$ shows that when the short-run fluctuation deviates from the long-run equilibrium,the close index of Shanghai (Shenzhen) market will be pulled back to equilibrium state by adjusting strength 0.00761(0.0038). It is obvious to see the adjusting strength of Shanghai market is more bigger than that of Shenzhen market; the second factor was the total effect of the four (three) term short-run fluctuation, in (7),they include the current, lag-one fluctuation of the turnover of Shanghai; the current, lag-one fluctuation of close index of Shenzhen. Four term coefficients: $0.0085,0.0023,0.691,-0.0282$ respectively reflect the effect strength size of short-run fluctuation. In (8), they include the current, lag-one fluctuation of the turnover of Shenzhen; the current fluctuation of close index of Shenzhen. Three term coefficients: 0.01, 0.0023, 0.551 respectively reflect the strength size of the effect of short-run fluctuation. In (7), it is obvious to see that the lag-one fluctuation of the close index of Shenzhen, $\Delta l y_{1 t-1}$ affects the fluctuation of the close index of Shanghai, coefficient -0.0282 shows the effect is the reverse,however, In (8),on the contrary, the lag-one fluctuation of the close index of Shanghai, $\Delta l x_{1 t-1}$ doesn't affect the fluctuation of the close index of Shenzhen.

In (7) and (8), $\Delta l x_{1 t}, \Delta l y_{1 t}$ also represent the daily return rate of Shanghai, Shenzhen stock market respectively, $\Delta l x_{2 t}, \Delta l y_{2 t}$ stand for the corresponding daily turnover variation rate of two karkets. In (7), $\Delta l x_{1 t}$ will change $0.0085(0.691)$ unit when $\Delta l x_{2 t}\left(\Delta l y_{1 t}\right)$ changes 1 unit but another three factors keep unchanged. $\Delta l x_{1 t}$ will change 0.0023 unit when $\Delta l x_{2 t-1}$ changes 1 unit but another three factors keep unchanged. $\Delta l x_{1 t}$ will decrease(increase ) 0.0282 unit when $\Delta l y_{1 t-1}$ increases(decreases) 1 unit but another three factors keep unchanged. In (8), $\Delta l y_{1 t}$ will change $0.01(0.551)$ unit when $\Delta l y_{2 t}\left(\Delta l x_{1 t}\right)$ changes 1 unit but another two factors keep unchanged. $\Delta l y_{1 t}$ will change 0.0023 unit when $\Delta l y_{2 t-1}$ changes 1 unit but another two factors keep unchanged.

\section{References}

Ai-mei Zhai, \& Tong Zhou. (2011). The Stock Market Price Volume Relation Analysis: Model and Empirical Tests. Technology Economy and Management Research, 11, 86-90.

Banerjee, A., Dolado, J. J., Galbraith, W., \& Hendry, D. F. (1993). Cointegration, Error Correction and the Econometric Analysis of Nonstationary Data. Oxford University Press, Oxford. http://dx.doi.org/10.1093/0198288107.001.0001

Engle, R. F., \& Granger, C.W.J. (1987). Cointegration and error correction: representation, estimation and testing. Econometrica, 55, 251-276. http://dx.doi.org/10.2307/1913236

Ghali, El-Sakka. (2004). Energy Use and Output Growth in Canada: A Multivariate Co-integration Analysis. Energy Economics, 26, 225-238. http://dx.doi.org/10.1016/S0140-9883(03)00056-2

Johansen, S. (1988). Statistical analysis of cointegration vectors. Journal of Economic Dynamics and Control, 12, 231-254. http://dx.doi.org/10.1016/0165-1889(88)90041-3

Jumbe, C. B. L. (2004). Co-integration and Causality between Electricity Consumption and GDP: Empirical Evidence from Malawi. Energy Economics, 26, 61-68. http://dx.doi.org/10.1016/S0140-9883(03)00058-6

Lee, C. (2005). Energy Consumption and GDP in Developing Countries: A Co-integrated Panel Analysis. Energy Economics, 27, 415-427. http://dx.doi.org/10.1016/j.eneco.2005.03.003

Liang Guo, \& Weixing Zhou. (2010). An Empirical Analysis of the Volume-Price Relation Using High-Frequency Data in the Chinese Stock Market. Chinese Journal of Management, 7(8), 1242-1247.

Menghua Tong, \& Chengming Wu. (2009). Empirical research on relation between volume and price based on the CARR model in Shanghai stock market. Journal of Liaoning Technical University(Social Science Edition) 11(1), 16-19.

Na Sun, \& Deshan Sun. (2012). The Comparison and Analysis of GARCH Model and The ECM Model in 
Predicting Shanghai Stock Index and Shenzhen Stock Index. Science \& Technology Vision, 3, 9-12.

Qiang Hou. (2009).The Relationship between Energy Consumption Growths and Economic Growth in China. International Journal of Economics and Finance, 1(2), 232-237.

Xiaoyan Zhou. (2012). Empirical Analysis of Relation between Price Change and Volume in Shenzhen Stock Market: Application of Economic Mathematics Model. Journal of Longyan University, 30(2),14-18.

Xiuhai Huang. (2012). Research on Dynamic Conduction Effect Between Economy and IPO Fund Based on Cointegration and Non- symmetric ECM. Research on Economics and Management, 2, 57-62. 